\title{
Imitating Simulation of Thermomechanical Processing of Metals
}

\section{${ }^{1}$ Akhmedov Akrom Burkhanovich, ${ }^{2}$ Grigorev Pavel Sergeevich, ${ }^{3}$ Ibodulloev Sherzod Rustamovich, ${ }^{4}$ Kuldibaevna Lutfiya Alijanovna}

${ }^{1}$ Professor, Department of Mechanics and mathematical modelling, National University of Uzbekistan named after Mirzo Ulugbek, Tashkent, Uzbekistan.

${ }^{2}$ Assistant Professor , Department of Electric Trains and Locomotives , Russian University of Transport (MIIT), Moscow, Russia.

${ }^{3}$ Phd student, Department of Mechanics and mathematical modelling, National University of Uzbekistan named after Mirzo Ulugbek, Tashkent, Uzbekistan.

Kuldibaevna Lutfiya Alijanovna

${ }^{4}$ Phd student, Department of Mechanics and Mathematical Modelling, National University of Uzbekistan named after Mirzo Ulugbek, Tashkent, Uzbekistan.

Email: sherzod_uzmu@inbox.ru

Article History: Received:11 January 2021; Accepted: 27 February 2021; Published online: 5 April 2021

\begin{abstract}
Thermomechanical processing of metals by cutting is a complicated technological problem that is difficult for mathematical simulation. The various phenomena observed in this process are so closely intertwined with each other and their interaction is so complex that eleven relatively independent theories not coming yet to a holistic unity are focused on the cutter edge. These are a theory of chip formation, metal cutting mechanics, a friction theory in metalworking, thermodynamics of cutting, a theory of wear and resistance of cutting tools.

A mesh-free method of Smoothed Particle Hydrodynamics (SPH) has been used for simulation in this paper. The SPH-based simulation in LS-DYNA is performed to predict cutting forces and plastic deformations for machining processing of metals by cutting. The results characterizing the distribution patterns of the strain tensor components and the temperature field at different points in time and space have been presented. The performed studies have demonstrated that it is possible to use changes in the temperature fields as a criterion for estimating the elastic-plastic deformations.
\end{abstract}

Keywords: Smoothed Particle Hydrodynamics (SPH), thermodynamic processe, experimental result, thermomechanical process, metal cutting simulation.

\section{Introduction}

Thermomechanical processing of metals has been used by man for a long time and will remain the most important production process now and in future as well. It is a set of deformation operations of heating and cooling (in different sequences) as a result of which a final structure of the metal alloy surface is formed. Therefore, even minor improvements in efficiency and effectiveness of any cutting operation have an influence on a huge amount of applications of this processing. For this reason, mathematical simulation is of great interest to both academia and industry.

Machining processing of metals by cutting is accompanied by thermodynamic processes. A source of heat when cutting metals is the work spent for plastic and elastic deformations in the cut layer and in the layers adjacent to the processed surface and the cut surface and for overcoming friction along the front and rear surfaces of the cutter. In the process of plastic deformation, the metal grains move relative to each other, which is an additional source of heat generation and heat release.

The heat released during the cutting process is not concentrated in the places of its formation, but, according to the laws of thermodynamics, spreads over the sample volume from points with a higher temperature to points with a lower one. In the process of metal cutting the chips and contact surfaces are heated within the temperature range $500-1000^{\circ} \mathrm{C}$. Approximately $95 \%$ of the deformation and friction energy are converted into heat that is mainly absorbed by chips $50-86 \%$, a cutter $10-40 \%$, a product under processing $3-9 \%$ and about $1 \%$ of the heat is radiated in surrounding area [1-3].

\section{Materials and Methods}

Due to the complex nature, the process of cutting solids is very difficult to study. The diverse phenomena considered here are so closely intertwined with each other and their interactions are so complex that eleven relatively independent theories, which have not yet come to an integral unity, are focused on the cutter edge. These are a theory of chip formation, the mechanics of metal cutting, a theory of friction in metalworking, thermodynamics of cutting, a theory of wear and resistance of cutting tools. The experimental and empirical studies [13-26] are used to understand the process of metal cutting. Analytical models are applied as well [1-11]. 
In particular, the methods based on Lagrangian particles are capable of trapping large material deformations because of the absence of meshes. In these methods there are no mesh-related problems such as distortion, entanglement. At present the popular method of metal cutting simulation is a smoothed particle one (SPH) [7-10].

SPH is a mesh-free method since it does not require a background mesh. In SPH, the computational area is represented by Lagrangian particles carrying alternating fields and moving with the material. The meshfree nature of SPH makes it an ideal candidate for imitating simulation of thermomechanical processing of metals. LS-DYNA SPH-based simulation is performed [11-13] to predict cutting forces, plastic deformations and cutting plane with sufficient accuracy.

The paper discusses the problems of machining processing of a metal sample in the form of a parallelepiped clamped from below. Let us consider two options of the problem statement: Option 1 -the remaining faces are free and Option 2 - one of the faces is clamped (Fig. 1).
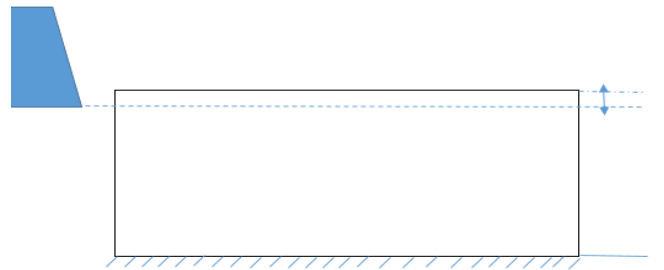

Option 1

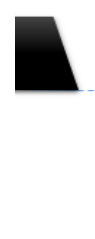

Fig. 1

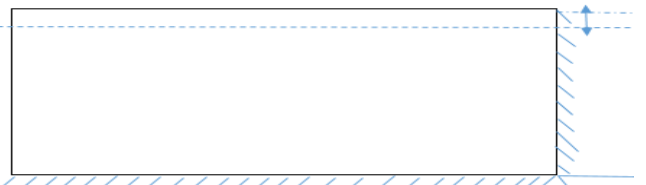

Option 2

For the simulation of the metal cutting process, a Johnson-Cook empirical model of flow stress [6-7] is used to describe the evolution of the fluidity limit dependent on a current level of plastic deformation. For the process of damage to be simulated, a cumulative model of damage is used [13-16]. For ductility and destruction the empirical method proposed by Johnson and Cook depends not only on the actual plastic deformation, but also on the strain rate and material temperature. The parameter values for the Johnson-Cook method are given in Table 1.

Table 1

The parameter values for the Johnson-Cook ductility and destruction model

\begin{tabular}{|c|c|c|}
\hline Parameter & Value & unit \\
\hline $\mathrm{A}$ & $5.53 \cdot 10^{8}$ & $\mathrm{~N} / \mathrm{m}^{2}$ \\
\hline $\mathrm{B}$ & $6 \cdot 10^{8}$ & $\mathrm{~N} / \mathrm{m}^{2}$ \\
\hline $\mathrm{C}$ & $1.34 \cdot 10^{-2}$ & - \\
\hline $\mathrm{n}$ & $2.34 \cdot 10^{-1}$ & - \\
\hline $\mathrm{m}$ & 1.00 & $K$ \\
\hline $\mathrm{T}_{\text {melt }}$ & $2,93,15$ & $K$ \\
\hline $\mathrm{T}_{\text {ambient }}$ & $1.00 \cdot 10^{-3}$ & $1 / \mathrm{s}$ \\
\hline$\dot{\varepsilon}_{\text {pl,0 }}$ & 0.06 & - \\
\hline $\mathrm{D}_{1}$ & 3.31 & - \\
\hline $\mathrm{D}_{2}$ & -1.96 & - \\
\hline $\mathrm{D}_{3}$ & $1.8 \cdot 10^{-3}$ & - \\
\hline $\mathrm{D}_{4}$ & 0.58 & - \\
\hline
\end{tabular}

\section{Results}

For the workpiece model (Table 1) of $12.58 \mathrm{~mm}$ long, $4.3 \mathrm{~mm}$ high and $4 \mathrm{~mm}$ wide the SPH particles are arranged in accordance with the object consisting of quadratic cells with an edge length of $0.06 \mathrm{~mm}$. All the particles have the initial ambient temperature; an adiabatic system is considered. To prevent the model from unrestricted translational motion, the lower row of SPH particles is at room temperature and is fixed to the base.

For simulation of the orthogonal cutting process the continuous chips are separated from the workpiece in Fig. 2. In this case, the depth of cutting is to $0.062 \mathrm{~mm}$ with the cutting rate of $1.6 \mathrm{~m} / \mathrm{c}$. The study of the equivalent strain distribution of Mises obtained from statically discredited model reveals that the highest level of strains marked by red color appears near the tip of the cutting tool where mainly a shear strains occur. The zones showing a high value of the equivalent strains are surrounded by those with the average level of strains (yellow and green colors). The SPH particles located at some distance both from the tool tip and from the basic zone of shear show the low values of strains (blue color). As a consequence, in the workpiece under study the high gradients of strains are in the region of the cutting edge of the tool. When looking at the results shown in Fig. 3, it is important to remember that the particles depicted are only interpolation points at which the SPH equations estimate a continuum of the model, not the region of a particular material. 

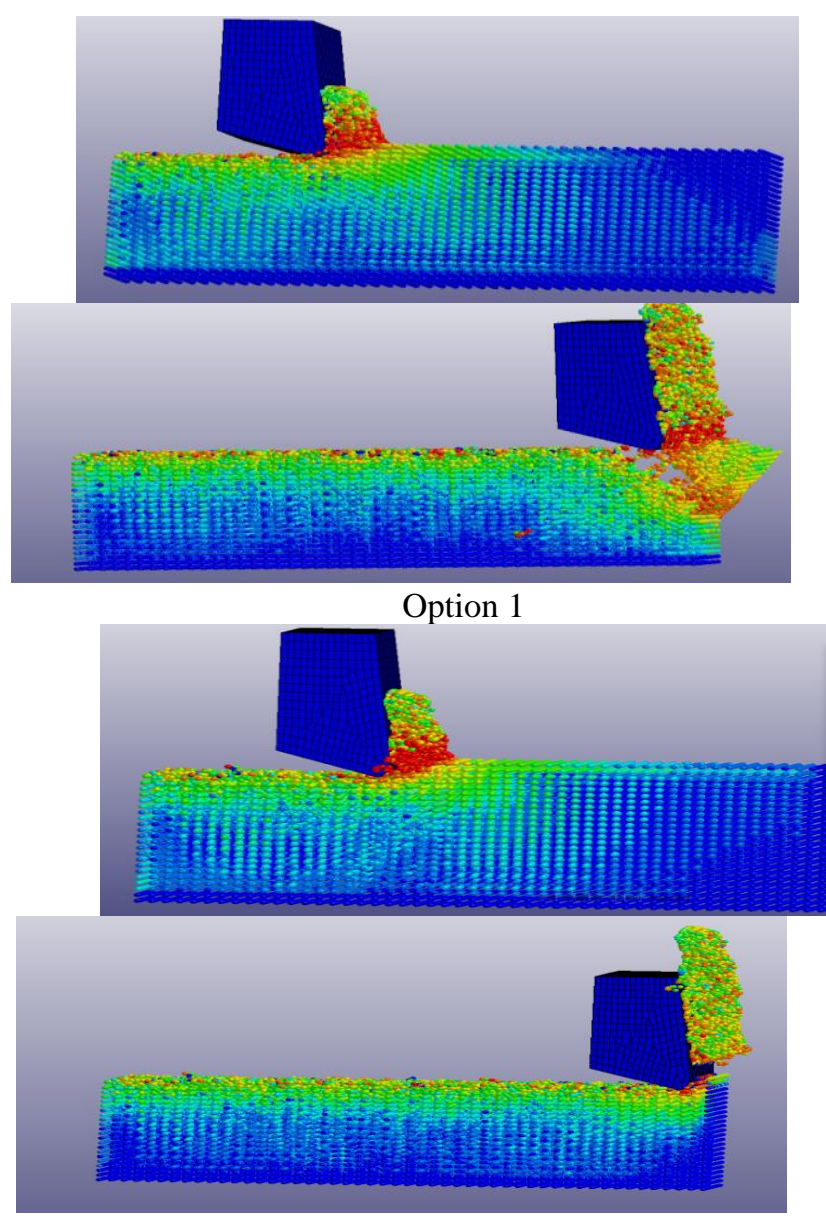

Option 2

Fig. 2. The distribution of strain intensity in an object (red - high strain; blue - low strain). The nature of deformation of material points of the processed sample are given for two cases of their fixation

From the results obtained one can see a clear example of the implementation of the Saint-Venan principle [10] stating that a degree of the influence of the sample fixation is not significant at sufficiently distant points. However, near the free edge, the action of the cutter leads to destructing the processed material, which is extremely undesirable for the smooth surfaces to be obtained after machining processing. Unlike Option 1 (Fig. 1), Option 2 achieves a sufficiently smooth surface, which should be taken into account for new technologies of metal processing to be developed.

In the process of simulation of the problem under consideration it was revealed that after a sudden impact of the cutter into the workpiece in the both cases of fixation there is a non-stationary distribution of the strain field due to the occurrence of wave processes. From the results obtained (Fig. 3) it follows that for Option 1 (Fig. 1) in the formed chip the strains of longitudinal compression arise and then a gradual decrease of them begins. For Option 2, the longitudinal strains of compression arise at the end points of the chip and the longitudinal strains of stretch in the middle of the chip.

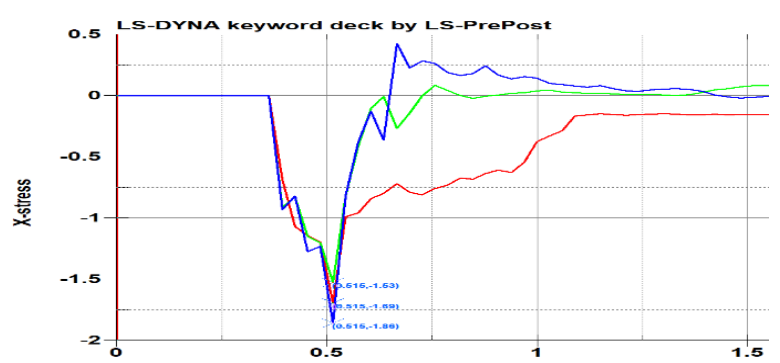

a)

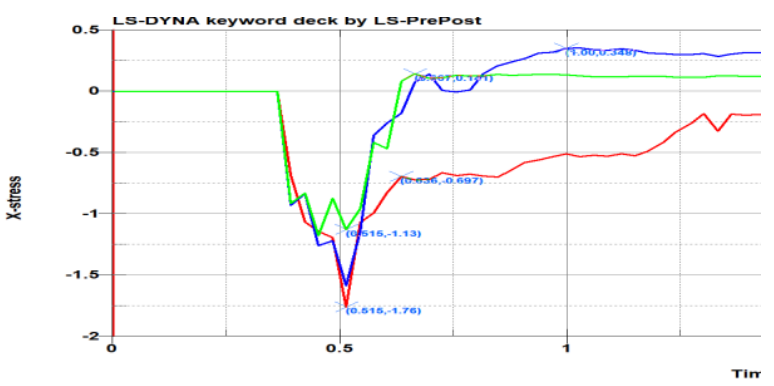

b) 
Fig. 3. A graph of the change in the longitudinal strain in the direction of cutting motion for two options of the fixation at different points along the line of the cutter action. The red line is the end point, the green line is the point located in one quarter, and the blue line is in the middle along the line of cutter action

Thus, it should be noted that the obtained results qualitatively coincide with the available data [17-23], which is evidence of an adequacy of the proposed computational algorithm for metal cutting.

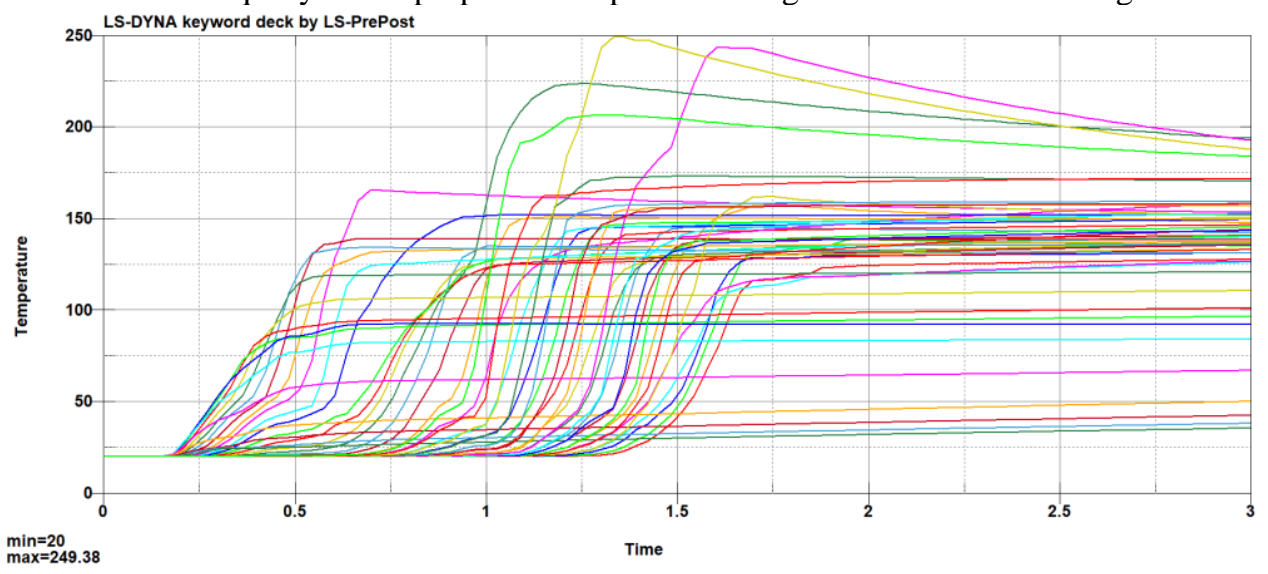

Fig. 4. The distributions of temperature in time in different elements located in the perpendicular directions and at different positions of the cutter

As it follows from the results obtained (Fig. 4), for the first position of the cutter on the second line of the first layer of the sample under consideration, a sharp increase in temperature is observed and then the temperature stabilizes with time; the highest temperature is in the center of the cross section. For the second and third positions of the cutter, due to the accumulation of temperature, a gradual increase in temperature is observed from a point to a point of cutter action, which has a tendency of the decrease in time, which is associated with the redistribution of the temperature field.

\section{Imitating simulation of rotating object cutting}

The processes of orthogonal cutting of metals occur by relative longitudinal displacement of a cutting tool and a workpiece. Such technologies serve as a main tool of surface cleaning from hard alloys. Here, the main problem is to provide cleanness of workpiece surface, which is particularly important, for example for drilling rigs. The most useful can be the simulation models of machining processing of various workpieces rotating around their central axis.

The SPH methodology for simulation and analysis of the three-dimensional machining process is used in this paper. The classical methods such as the finite element method (FEM) cannot overcome large distortions for the processes of finite elastic-plastic deformations. Of course, the elemental analysis of the metal cutting processes does not often work due to a strong deformation of the mesh.

The orthogonal cutting process for AISI H13 steel was simulated and analyzed by the SPH method. The developed SPH model made it possible to correctly estimate the cutting forces, as shown in two orthogonal cutting situations. For cutting forces, the numerical and experimental results were compared.

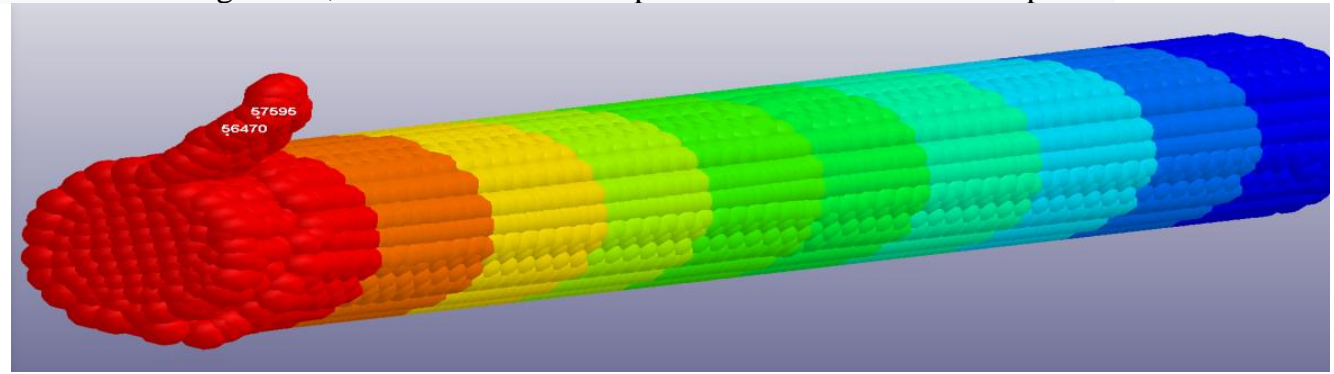

Fig. 5. The nature of the change in the stress-strain state in the process of chip formation during machining processing by cutting of cylindrical metal rods at different distances from the cutter

From the results obtained (Fig. 5) it follows that in the nodes closest to the cutting tool there is a large gradient of the distribution of the stress-strain state, this gradient disappears gradually with the distance along the cylinder.

In the initial stage of machining processing of cylindrical samples (Fig. 6), due to sudden impact of the cutting tool, there is a stepwise increase in the field of temperatures and residual finite strains, which is evidence of the complex stress-strain state in the initial stage of chip formation. 


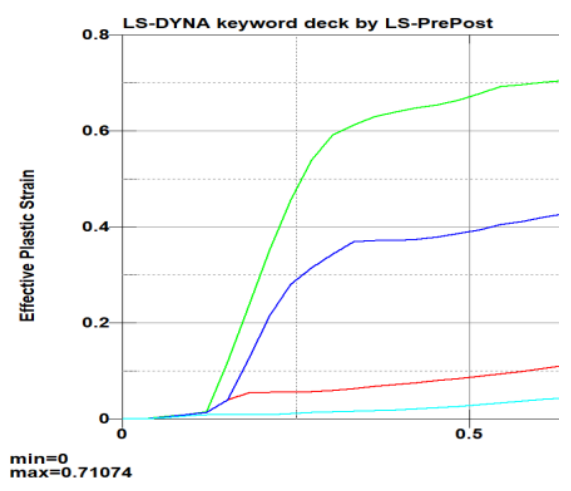

a)

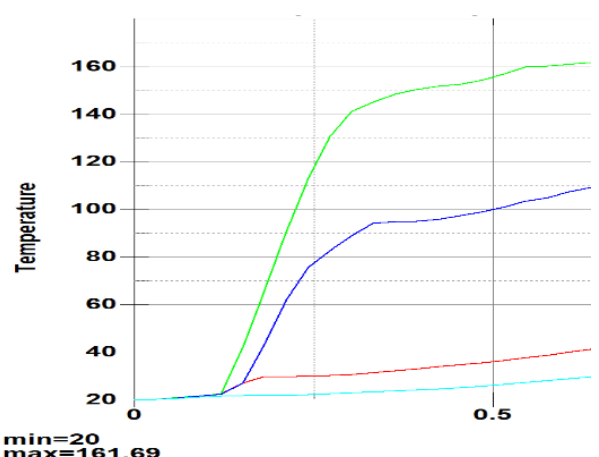

b)

Fig. 6. The graphs of changes in the intensity of final elastic-plastic deformations: a) and the temperature field; $b$ ) in the process of chip formation during machining processing of cylindrical workpieces

On the other hand, a synchronous almost identical dependence on time is observed between the temperature distribution and final elastic-plastic deformations. As a result, it becomes possible to accept the change in the temperature field as a criterion for estimating the emerging elastic-plastic deformations.

\section{Conclusions}

Numerical simulation of machining processing of metals such as metal cutting is a complex problem in the mechanics of deformable solid. These processes include plastic deformation localization, chip-formation, high-gradient temperature, leading to structural changes in the samples under consideration. For imitating simulation of machining processing of metals by cutting the SPH-particle method has been used in this paper. The SPH particles in their nature are capable of trapping large plastic deformation of a material. The results obtained show an almost proportional dependence between temperature field changes and elastic-plastic deformations. However, the SPH method is instable relative to stretching, which leads to instability of calculations. Hence, the method requires some modification.

The use of the SPH method for machining processing of metals is quite new; its features have not been fully understood, and the most effective ways of its application have not still found completely. Despite its novelty, the SPH method can be considered as a very promising tool for studying the processes of machining cutting of metals and chip formation.

\section{References}

1. Bert Verlinden,Julian Driver et al Thermo-Mechanical Processing of metallic Materials, ISBN 97800805444970 //Elsiver Science 2007.

2. Monaghan J.J., 2005. Smoothed Particle Hydrodynamics, Reports on Progress in Physics, 68(8):17031759.

3. Gingold R.A., Monaghan J.J., 1977. Smoothed Particle Hydrodynamics: Theory and application to non-spherical stars, Monthly Notices of the Royal Astronomical Society, 181:375-389.

4. Toshmatov E., Akhmedov A., Thermodynamic bases of mechanical working of metals by cutting.// E Toshmatov et al 2020 IOP Conf.Ser.:Mater.Sci.Eng. $883 \quad 012098$ doi.org/10.1088/1757899X/883/1/012098.

5. Liu M.B., Liu G.R., 2010. Smoothed Particle Hydrodynamics (SPH): An overview and recent developments, Archives of Computational Methods in Engineering, 17(1):25-76.

6. Spreng F., Eberhard P., 2013. The way to an enhanced Smoothed Particle Hydrodynamics formulation suitable for machining process simulations, Proceedings of the 8th International SPHERIC Workshop.

7. Johnson G.R., Cook W.H., 1983. A constitutive model and data for metals subjected to large strains, high strain rates and high temperatures, Proceedings of the 7th International Symposium on Ballistics, 547:541-547.

8. Johnson G.R., Cook W.H., 1985. Fracture characteristics of three metals subjected to various strains, strain rates, temperatures and pressures, Engineering Fracture Mechanics, 21(1):31-48.

9. Benz W., Asphaug E., 1994. Impact simulations with fracture. I. Method and tests, Icarus, 107(1):98116.

10. Khojibergenov D T, Khozhibergenov U D, Akhmedov A B (2019) Some aspect of the construction of a mathematical model for cutting metals. News Natl. Acad.Sci. Ser. Geol.Tech.Sci. doi:10.32014/2019.2518-170X.40.

11. Muller M., Schirm S., Teschner M., Heidelberger B., Gross M., 2004. Interaction of fluids with deformable solids, Computer Animation and Virtual Worlds, 15(3-4):159-171. 
12. Boothroyd G., Knight W.A., 2006. Fundamentals of Machining and Machine Tools, 3rd edition, Boca Raton: CRC.

13. Cleary P.W., Monaghan J.J., 1999. Conduction modelling using Smoothed Particle Hydrodynamics, Journal of Computational Physics, 148(1):227-264.

14. Spreng F., Schnabel D., Mueller A., Eberhard P., 2014. A local adaptive discretization algorithm for Smoothed Particle Hydrodynamics, Computational Particle Mechanics, 1(2):131-145.

15. Gaugele T., Eberhard P., 2013. Simulation of cutting processes using mesh-free Lagrangian particle methods, Computational Mechanics, 51(3):261-278.

16. Armarego E.J.A., Brown R.H., 1969. The Machining of Metals, Engle-wood Cliffs: Prentice-Hall.

17. Libersky L. D., Petschek A. G., Carney T. C., Hipp J. R., Allahdadi F. A., High strain lagrangian hydrodynamics: A three-dimensional SPH code for dynamic material response, Journal of Computational Physics 109 (1993) 67-75.

18. Liu M. B., Liu G. R., Smoothed particle hydrodynamics SPH: an overview and recent developments, Archives of Computational Methods in Engineering 17 (1) (2010) 25-76.

19. Vuyst T. De, Vignjevic R., Total lagrangian SPH modelling of necking and fracture in electromagnetically driven rings, International Journal of Fracture 180 (1) (2013) 53-70.

20. Chakraborty S., Shaw A., A pseudo-spring based fracture model for SPH simulation of impact dynamics, International Journal of Impact Engineering 58 (2013) 84-95.

21. Campbell J., Vignjevic R., Libersky L., A contact algorithm for smoothed particle hydrodynamics, Computer methods in applied mechanics and engineering 184 (1) (2000) 49-65.

22. Belytschko T., Yeh I., The splitting pinball method for contact-impact problems, Computer methods in applied mechanics and engineering 105 (3) (1993) 375-393.

23. Batra R. C., Zhang G. M., SSPH basis functions for meshless methods, and comparison of solutions with strong and weak formulations, Computational Mechanics 41 (4) (2008) 527-545.

24. Leroch S., Varga M., Eder S., Vernes A., Ripoll M. R., Ganzenmüller G., Smooth particle hydrodynamics simulation of damage induced by a spherical indenter scratching a viscoplastic material, International Journal of Solids and Structures 81 (2016) 188-202.

25. House J. W., Lewis J. C., Gillis P. P., Wilson L., Estimation of flow stress under high rate plastic deformation, International journal of impact engineering 16 (2) (1995) 189-200.

26. Bespalko et al 2020 IOP Conf. Ser.: Mater. Sci. Eng. 883012134 doi.org/10.1088/1757899X/883/1/012134. 